\title{
Jacques Ellul : l'homme de tous les combats
}

Entrevue avec Dominique North Ellul et réalisé par Christian Mesnil

\section{Christian Mesnil}

\section{CpenEdition}

\section{Journals}

Édition électronique

URL : http://journals.openedition.org/communicationorganisation/1793

DOI : 10.4000/communicationorganisation. 1793

ISSN : 1775-3546

Éditeur

Presses universitaires de Bordeaux

\section{Édition imprimée}

Date de publication : 1 mai 1995

ISSN : 1168-5549

\section{Référence électronique}

Christian Mesnil, « Jacques Ellul : I'homme de tous les combats », Communication et organisation [En

ligne], 7| 1995, mis en ligne le 26 mars 2012, consulté le 03 mai 2019. URL : http://

journals.openedition.org/communicationorganisation/1793 ; DOI : 10.4000/

communicationorganisation. 1793

Ce document a été généré automatiquement le 3 mai 2019.

(c) Presses universitaires de Bordeaux 


\title{
Jacques Ellul : l'homme de tous les combats
}

\author{
Entrevue avec Dominique North Ellul et réalisé par Christian Mesnil
}

Christian Mesnil

1 - Le 19 mai 1994 nous quittait Jacques ELLUL, à l'âge de 82 ans. Il avait deux garçons : Jean et Yves et une fille, vous même, qui habitez actuellement Castres avec votre mari et votre fils tout en exerçant la profession de skipper. Je vous remercie d'avoir accepté cet entretien qui n'a pas d'autre ambition que d'atteindre les deux objectifs suivants. D'une part rappeler la démarche de votre père pour donner à ceux qui le connaissent mal l'envie d'approfondir ses ouvrages. D'autre part proposer aux spécialistes qui ont côtoyé votre père ou étudié son œuvre un témoignage particulier et inhabituel.

Comment présenteriez-vous à nos lecteurs ce qu'il a été et ce qu'il a réalisé ?

3 Dominique NORTH-ELLUL :

4 - Mon père est né en 1912 à Bordeaux. Il a été avant guerre chargé de cours à la faculté de Droit de Montpellier puis de Strasbourg. Mais, pour avoir soutenu devant un petit groupe d'étudiants des opinions anti nazis, le gouvernement de Vichy le révoque. Dans les années cinquante, il se consacre à ses livres et à son travail universitaire mais aussi à des engagements forts comme l'Eglise Réformée de France, les Clubs de prévention de la délinquance juvénile (1958 à 1977), et le combat écologique en tant que co-fondateur du Comité de Défense de la Côte Aquitaine avec son ami Bernard CHARBONNEAU.

Compte tenu de ses fonctions universitaires, s'il a été considéré comme un juriste c'était bien à tort car il se refusait à être un défenseur du Droit, il était un enseignant de certaines spécialités du Droit pour lesquelles il se passionnait, en particulier le Droit Romain, l'Histoire des institutions, le Marxisme et l'Histoire de la technique parce qu'il pensait qu'à partir de la compréhension de ces points-là on pouvait arriver à l'essentiel des mouvements sociaux et des grands schémas de la pensées humaine moderne. En ce qui concerne le Marxisme, certainement bien peu de Marxistes pourraient se vanter d'avoir lu l'intégralité des œuvres de Marx! Il faut dire que son cours avait une certaine réputation auprès des étudiants. Dans tous les cas de figures, l'aspect purement juridique 
et ces disciplines n'étaient pas son premier souci et il était plus un historien et un sociologue qu'un juriste.

6 Cela d'ailleurs exaspérait les gens car il était difficilement classable ; moraliste de gauche à relents d'anarchistes, théologien Barthien soutenant Israël et un certain nombre de valeurs dites de Droite: Tout cela faisait un cocktail qui irritait fort ses concurrents de Droite non-chrétiens tel Raymond ARON et ses amis de gauche de l'Eglise Réformée qui trempaient dans tous les bains à la mode. Néanmoins il répondait au questionnement existentiel de la Diaspora silencieuse et anonyme, juifs, catholiques, athées, objecteurs de consciences et soixante-huitards de tous les bords, s'y retrouvaient, frères d'un dialogue vrai, grâce à la pertinence de ses analyses et c'est cela qui lui importait. Finalement ses ouvrages qui se montent à un cinquantaine de bouquins et quelques mille articles ont été beaucoup mieux acceptés et compris à l'étranger.

7 Ch. M. : - Parmi les caractéristiques mises en avant par ceux qui ont eu la chance de le connaître reviennent souvent trois éléments: sa rigueur intellectuelle, son attitude profondément modeste et un peu secrète et la qualité d'écoute à la fois attentive et affectueuse dont il enveloppait ses interlocuteurs. Qu'en pensez-vous?

8 D. N-E. : - Au niveau de la rigueur, c'est tout à fait évident. Pour lui la rigueur, était un moyen qui lui permettait d'être crédible. Toutes ses théories ne pouvaient que susciter des heurts, des critiques ou des révolutions. Il fallait absolument qu'il soit rigoureux, qu'il ait des arguments solides. Il s'entourait de toutes les informations publiées avant de rédiger le moindre article. Il était une référence pour beaucoup d'entre nous car il avait toujours une réponse fiable.

9 C'était aussi effectivement un homme très simple dans sa vie de tous les jours, sa façon de s'habiller, son attitude envers les autres quelle que soit leur place dans la société, et modeste par rapport à son œuvre car il ne se situait jamais en termes de réussite sociale ou de supériorité... Son attitude d'écoute était souvent une attitude d'aide. Beaucoup de gens venaient lui demander son avis sur des sujets difficiles, personnels ou professionnels, même des grands de ce monde. Il n'a jamais été intimidant et c'était bien dans sa ligne d'être à la fois à la portée des «petits » et en lien avec les "grands » qui peuvent jouer un rôle précieux pour faire évoluer la société.

Ch. M. : - Y-a-t-il d'autres traits de son caractère que vous souhaiteriez souligner?

11 D. N-E. : - Oui, son courage ! C'était un homme de cœur qui a été de tous les combats qui se sont trouvés sur son chemin. A ce courage, il faut ajouter un don de prophétie dont plusieurs interlocuteurs ou lecteurs ont parlé à son sujet.

Ch. M. : - Dans quelles circonstances par exemple?

D. N-E. : - Elles sont nombreuses. Je citerais le communisme que ce soit en Union Soviétique, en Chine ou au Cambodge. Je me souviens d'une discussion entre mon frère aîné qui habitait le Cambodge et mon père. Mon frère soutenait que dans le Cambodge, pays de la fantaisie et de la joie de vivre, ne pouvait s'instaurer qu'un communisme doux. Mon père était d'un avis contraire bien avant que la tragédie qu'a connue ce pays ne lui donne raison.

14 Il a également été prophétique bien sûr à propos de la technique, son premier livre « La Technique ou l'enjeu du siècle » remonte à 1953. II l'a également été à propos de l'Islam, à propos des mouvements politiques français et de l'écologie. 
Ch. M. : - Y-a-t-il dans sa vie des faits lourds de conséquences qui expliqueraient certaines positions ou attitudes?

D. N-E. : - Il y a eu d'abord la résistance.

Un grand nombre de résistants l'ont été par rigueur morale mais aussi par obligation. Mon père était de ceux-là non seulement à cause de sa clairvoyance, mais, aussi parce que son père avait été prisonnier et que sa femme était recherchée en tant qu'étrangère. Mes parents ont été résistants pour se sauver eux-mêmes mais aussi parce qu'ils pensaient qu'en tant que chrétiens leur devoir était de sauver des Juifs et toutes personnes en danger. Dans leur petit coin de Gironde, en pleine campagne, ils servaient de relais pour faire transiter les gens vers l'Espagne. Mais cela s'est payé d'un énorme traumatisme et d'une accumulation terrible d'angoisses dont il ne parlait pas. En tout cas il n'a jamais oublié cette époque.

Ch. M. : - Est-ce le seul point que vous souhaitez rappeler ou dévoiler?

D. N.-E. : - A la fin de sa vie un second traumatisme a été l'abandon de l'église réformée locale. Toute sa vie il a eu l'amour de son église. Une fois de plus on s'est trompé sur lui. Il était à la fois le héros, le grand Ellul et la bête à abattre. On n'a pas compris qu'il se battait pour la paroisse de Pessac qu'il avait créée et l'église reformée de Bordeaux a été inommable.

On ne se rend pas compte de sa souffrance vers la fin de sa vie.

Ceux qui n'ont pas eu le courage de l'affronter lorsqu'il était en pleine possession de ses moyens ont profité ensuite de sa faiblesse. Alphonse MAILLOT évoque cette situation dans le numéro spécial de Foi et Vie qui vient de paraître et écrit à propos des instances de l'Eglise Réformée «C'est triste car elles n'ont pas su utiliser un tel génie ».

Ch. M. : - Pourquoi n'a-t-il jamais voulu quitter la région bordelaise?

D. N-E. : - Il y a d'abord le sens des racines et de la fidélité je crois. Il y a le terroir, cette côte aquitaine qu'il a défendue constamment. Il y a enfin l'antiparisianisme et le refus des honneurs.

Il exécrait absolument les modes liées aux gourous parisiens et à l'intelligentsia. Il n'aimait pas paraître à la télévision ou dans des cocktails. Il a eu un livre passé au pilon par son éditeur car il avait refusé d'aller faire le guignol à la télévision. Il estimait qu'il pouvait faire passer son message autrement.

Ch. M. : - Pourquoi cette méfiance vis à vis de la télévision?

D. N-E. : - Tel que cela était fait, cela ne lui plaisait pas. Et puis, cela signifiait des compromissions. Ce n'était pas seulement la télévision, mais tout ce qui était autour. Pour défendre la côte aquitaine par exemple il a employé les médias, mais pour se faire valoir lui-même, non! II y a une chose qu'il a regrettée par contre, c'est l'Académie. Il n'était pas pour les honneurs mais l'Académie, c'était un peu différent par nature. Il l'a refusée parce que cela entraînait de plus fréquents déplacements à Paris mais aussi...à cause de sa femme qui lui a dit: «Tu ne vas pas aller faire l'andouille, tu ne vas pas te faire valoir comme tout ce beau monde!»

Ch. M. : - Pouvez-vous nous parler du rôle que votre mère a joué auprès de son mari ?

D. N-E. :- Elle était plus intransigeante que lui sur le plan des honneurs. Elle ne souhaitait pas qu'il conquière la gloire. Ils ont fait ensemble un chemin commun je pense. Ils avaient tous deux cette aristocratie de l'âme qui consiste effectivement à ne pas s'agenouiller 
devant les puissants et à ne pas accepter les compromissions. Ils avaient le même tempérament pour cela. Dans les choix importants, les grandes options, elle avait son mot à dire.

Ch. M. : - Dans sa façon d'être que vous a-t-il transmis que vous accepteriez de partager avec les lecteurs de notre revue?

D. N-E. : - Le point le plus voyant, c'était son non conformisme. Il est issu d'un milieu qui l'a beaucoup poussé à ça parce que c'était un milieu artiste et désargenté chroniquement. Sa mère était peintre et faisait vivre le ménage par des rentrées d'argent irrégulières. Son père au caractère très entier se faisait éjecter de partout où il était. Tous les deux étaient des originaux. Le non conformisme de leurs fils n'était pas une attitude délibérée. Il était plutôt hors conformité. Il était aussi très rieur, très joueur, très désordonné. Son bureau était un vrai champ de bataille. Pour des gamins, c'était amusant : ça dégringolait de partout, il y avait des bouquins sur tous les murs et cinquante centimètres de papier par terre, comme un énorme tapis: le courrier, les choses inutiles... Il ne supportait évidemment pas qu'on vienne ranger tout ça.

Ch. M. : - N'est ce pas à mettre en parallèle avec le foisonnement de sa pensée ?

D. N-E. : - Exactement. Les choses importantes étaient toutes dans des dossiers bien rangés. Il était à la fois d'un désordre fabuleux et d'une rigueur et discipline extrêmes. Notamment pour son rythme de travail, il avait une régularité de métronome. Ce qu'il nous a apporté par ailleurs, c'est son ouverture à toutes les populations. A la maison on a vu passer toutes sortes de gens : les anarchistes, les hippies, les blousons noirs mais aussi des personnalités importantes de différents pays. Il était au service de chacun dès qu'il y avait un apport à faire mais cela ne l'empêchait pas d'aimer la solitude.

Enfin sa pensée n'était jamais abstraite. Elle était toujours reliée à des problèmes du jour. C'était l'homme des solutions.

Ch. M.: -Y-a-t-il eu dans la pensée de votre père des constantes dont vous pourriez témoigner?

D. N-E. : - Le cheminement de sa pensée s'organise autour de quatre pôles. Il avait un grand projet humaniste, des règles morales très strictes, un cerveau à nul autre pareil et un tempérament de guerrier.

Ch. M. : - Pourriez-vous détailler davantage ces quatre éléments?

D. N-E. : - 1 - Dans son grand projet humaniste il avait deux soucis principaux. D'abord trouver le message qui convient pour aider une société malade (analyse globale) et ensuite aider chacun à trouver les outils qui lui sont personnels et à redonner à sa vie un sens que cette société lui a enlevé (solutions concrètes). Je pense qu'il a eu très jeune ce grand projet et cette préoccupation d'assortir sa réflexion de propositions d'actions. $C$ est d'ailleurs ce qui l'a séparé du Mouvement Personnaliste de la Revue «Esprit » fondée par Emmanuel MOUNIER en 1934. Il a jugé que si ces idées n'étaient pas mises en pratique, Esprit ne serait qu'une revue de salon. Il a créé alors des petites communautés d'étudiants ayant pour objectif de transformer le milieu en développant la réflexion socio-politique à la base.

$38-2$ - En ce qui concerne les règles morales, il les avait aussi en lui. Elles tiennent d'abord à son caractère. C'est quelqu'un qui n'est jamais tombé dans la complaisance, qui n'admettait jamais les idées toutes faites, qui ne recherchait ni la réussite, ni les honneurs, qui ne voulait tomber dans aucun parti ni créer aucune école. 
39 Son combat consistait d'une part à combattre les idoles et les modes, d'autre part à défendre l'opprimé et le faible.

Au colloque qui lui a été consacré à Bordeaux l'an dernier, il a évoqué lui même en ces termes le sens de l'honneur que lui avait légué son père: «Cette notion périmée dans laquelle j'ai été formé comportait quatre règles : ne jamais mentir aux autres, ne jamais se mentir à soi-même, être miséricordieux envers les faibles, être inflexible devant les puissants $»$.

41 - 3 - Il y avait chez lui une capacité de travail absolument extraordinaire et un cerveau sans cesse en action. Il dormait très peu et ses heures d'éveil étaient consacrées à l'écriture.

42 - 4 - Enfin son tempérament guerrier s'est constamment manifesté par son besoin de démontrer, d'encourager les gens, de se battre pour faire avancer les choses à quelque niveau que l'on se trouve.

Ch. M. : - Quelles étaient pour lui les principales causes de cette maladie de notre société ?

D. N-E. : - Mon père en retenait essentiellement quatre en lien avec son éthique.

La première c'est la domination de l'argent. Il avait un mode de vie très simple et il donnait facilement son argent à son église et aux personnes dans le besoin, il ne comptait pas et avait horreur de l'esprit capitaliste. Enfin je puis dire qu'il a élevé ses enfants de la manière la plus spartiate possible afin qu'ils ne s'habituassent points aux tiédeurs du confort bourgeois ! Ce en quoi il n'a pas trop mal réussi !

La deuxième, c'est la politique, source de tricherie, de forfanterie et de bluff. Il n'a fait qu'un court passage au conseil municipal de Bordeaux après la guerre. Dés qu'il s'est aperçu qu'il fallait signer des dossiers qu'il n'avait pas le temps d'étudier à fond, il a préféré démissionner. La politique, ce sont toujours des faux problèmes, là n'était pas la vérité selon lui. A partir du moment où l'on s'engage politiquement, il faut être lucide. Le troisième c'est l'idéologie technicienne qui gangrène tout ce qu'elle touche. En particulier le goût de la puissance qui est inné chez l'homme (La Tour de Babel, c'est une vieille histoire) est démultiplié par le mythe de l'efficacité absolue induit par la technologie, par l'idéologie qui en découle et l'implacable logique de cette idéologie. L'esclavage humain y est toujours présent mais de manière plus cachée et plus perverse ainsi que les clivages sociaux, rien n'est résolu. Mon père pensait qu'il faut en priorité rendre sa lucidité à l'homme et sa capacité d'analyse. Enfin le quatrième ce sont l'état et la bureaucratie qui eux sont aussi des facteurs d'asservissement de l'homme par l'homme. Ce thème a été largement étudié par Bernard Charbonneau avec lequel dès l'âge de 20 ans il s'était partagé le travail concernant l'analyse globale de notre société ! C'était, vous le voyez, un projet de longue haleine!

Ch. M. : - Je suppose que vous arrivez maintenant à la deuxième phase de son projet, c'est à dire aux solutions?

D. N-E. : - Oui ! Par rapport à ce constat dramatique mon père a passé aux yeux de beaucoup comme le grand pessimiste. En fait les solutions qu'il préconise ont été très bien identifiées par Patrick CHASTENET dans son livre « Lire Ellul » qui est le modèle du genre et je me bornerai donc à reprendre purement et simplement ce qu'il a écrit là-dessus : plusieurs pistes s'imposent à nous :

50 - D’abord démystifier la puissance politique et désidéologiser la technique. 
51 - Ensuite ne pas se faire d'illusion sur notre démocratie qui sera une fausse démocratie tout le temps que les pouvoirs ne seront pas limités, le politique relativisé, les minorités respectées. Il préconisait de donner la priorité aux petits, d'accepter le pluralisme des opinions et de faire un choix délibéré de la non puissance.

52 - Puis égaliser le travail et les revenus, créer de petites unités de production, produire localement l'énergie, réduire le temps de travail et le salariat.

53 - Par ailleurs aider le tiers monde, réduire notre consommation, notre niveau de vie et notre croissance.

54 - Enfin faire disparaître l'armée et l'état centralisé.

55 Ch. M. : - Après le pessimisme de son analyse, je suppose qu'on a dû taxer ses propositions d'utopiques, d'irréalisables. Pourquoi préconisait-il de telles options à contrario de ce qui se fait actuellement?

D. N-E. : - Au fil de toutes ces solutions, on retrouve la foi du croyant, et un certain optimisme théologique.

Il y a une grande inconnue dans tout ça mais il parle en tant que chrétien : c'est l'inconnu de la transcendance qui doit intervenir. Il faut laisser à Dieu la possibilité de dire son mot... Il y a les solutions qu'il préconise pour l'homme, ce qu'il peut faire sur le plan politique et social qui a une petite couleur d'anarchie, et la foi qui est d'un autre ordre, la croyance en l'intervention de Dieu.. Pour lui si ce Dieu n'avait pas été de chair et ne pouvait pas nous aider dans nos combats quotidiens, il ne servait à rien. La Bible est toujours source d'anarchie, elle vomit sur les tièdes et préconise des positions renouvelées. Quand il parlait de "son" église reformée c'était toujours Reformata Semper Reformanda. Il refusait le titre de philosophe et plus encore celui de mystique. Le mysticisme, c'était l'horreur, ce qu'il y avait de pire au monde car il ne servait à rien.

Pour mon père, en bref, il ne fallait jamais s'asseoir nulle part dans une position confortable et ne jamais hurler avec les loups... 